МНОЖЕСТВЕННАЯ ГОРМОНАЛЬНАЯ РЕЗИСТЕНТНОСТЬ И МЕТАБОЛИЧЕСКИЕ НАРУШЕНИЯ ПРИ ПСЕВДОГИПОПАРАТИРЕОЗЕ

( ) Л.К. Дзеранова* , Н.В. Маказан', Е.А. Пигарова' , А.Н. Тюлякова' , Е.В. Артемова' , Т.В. Солдатова' , Д.О. Тулупов², А.В. Воронцов ${ }^{1}$, Г.А. Мельниченко

'ФГБУ «Национальный медицинский исследовательский центр эндокринологии» Минздрава России, Москва, Россия ФГБОУ ВО Российский национальный исследовательский медицинский университет им. Н.И. Пирогова Минздрава России, Москва, Россия

Псевдогипопаратиреоз - редкая группа клинически и генетически гетерогенных заболеваний, обусловленная инактивацией ПТГ-сигнального пути. Основным компонентом заболевания является резистентность к паратгормону (ПТГ), обуславливающая нарушение кальций-фосфорного обмена. При псевдогипопаратиреозе также могут отмечаться развитие нечувствительности к тиреотропному и гонадотропным гормонам гипофиза и формирование характерных клинических особенностей в виде подкожных кальцинатов, брахидактилии, ожирения, низкорослости, умственной отсталости. В данной статье приводится описание клинического случая псевдогипопаратиреоза у женщины 35 лет с классическим фенотипом гипопаратиреоза с наследственной остеодистрофией Олбрайта и доказанной мутацией в гене GNAS, обсуждается спектр метаболических расстройств, характерных для данного заболевания.

КЛЮЧЕВЫЕ СЛОВА: псевдогипопаратиреоз, ожирение, синдром Фара, паратгормон, гипотиреоз, кальций, гипокальциемия.

\title{
MULTIPLE HORMONAL RESISTANCE AND METABOLIC DISORDERS IN PSEUDOGYPOPARATIOSIS
}

(C) Larisa K. Dzeranova1*, Nadezhda V. Makazan', Ekaterina A. Pigarova1, Anna N. Tyulyakova', Ekaterina V. Artemova1, Tatiana V. Soldatova' ${ }^{1}$, Denis O. Tulupov ${ }^{2}$, Aleksandr V. Vorontsov, ${ }^{1}$ Galina A. Melnichenko',

'Endocrinology Research Centre, Moscow, Russia

${ }^{2}$ Pirogov Russian National Research Medical University, Moscow, Russia

Pseudohypoparathyroidism is a rare group of clinically and genetically heterogeneous diseases caused by the inactivation of the PTH-signaling pathway. The main component of the disease is resistance to PTH, causing a disturbance of calcium-phosphorus metabolism. With pseudohypoparathyroidism, there may also be a development of insensitivity to thyrotropic and gonadotropic hormones of the pituitary gland and the formation of characteristic clinical features in the form of subcutaneous calcifications, brachidactyly, obesity, stuntedness, mental retardation. This article describes the clinical case of pseudohypoparathyroidism in a 35-year-old woman with classic phenotypic hypoparathyroidism with hereditary Albright osteodystrophy and a proven mutation in the GNAS gene, and discusses the spectrum of metabolic disorders of the disease.

KEYWORDS: pseudohypoparathyroidism, obesity, Farah syndrome, parathyroidhormone, hypothyroidism, calcium, hypocalcemia.

\section{АКТУАЛЬНОСТЬ}

Среди редких причин множественных метаболических нарушений особое место занимает псевдогипопаратиреоз (ПГП), характеризующийся нечувствительностью к паратгормону (ПТГ) и возможностью формирования мультигормональной резистентности и наследственной остеодистрофии Олбрайта (НОО) [1]. Наследственная остеодистрофия Олбрайта объединяет в себе ряд характерных фенотипических особенностей: ожирение, подкожные кальцинаты, брахидактилия типа Е, низкорослость, умственная отсталость, лунообразное лицо. Гормональная резистентность может быть представлена сочетанием нечувствительности к ПТГ с резистентностью к тиреотропному гормону (ТТГ), гормон роста - рилизинг гормону (ГР-РГ), лютеинизирующему/фолликулостимулирующему гормонам (ЛГ/ФСГ).

В основе заболевания лежат инактивирующие дефекты на пострецепторном уровне внутриклеточной передачи сигнала. Механизмы внутриклеточной передачи сигнала от трансмембранного рецептора универсальны для множества пептидных гормонов и ряда других лигандов, чем и обусловлен мультисистемный характер нарушений, развивающихся при ПГП. Среди спектра молекулярно-генетических дефектов, ведущих к ПГП, наиболее распространенной и изученной является патология стимулирующей альфа-субъединицы G-белка (Gas). Особенности экспрессии гена GNAS, кодирующего Gas, обусловливают широкий клинический полиморфизм с неспецифическими вариантами манифестации и отсутствие четкой генотип-фенотипической корреляции. Псевдогипопаратиреоз - очень редкая патология, в связи с чем каждый случай метаболических нарушений, связанных с патологией в гене GNAS, представляет особый интерес.

\section{ОПИСАНИЕ СЛУЧАЯ}

В ФГБУ НМИЦ эндокринологии наблюдается женщина 35 лет с ожирением, низкорослостью, депрессивны- 


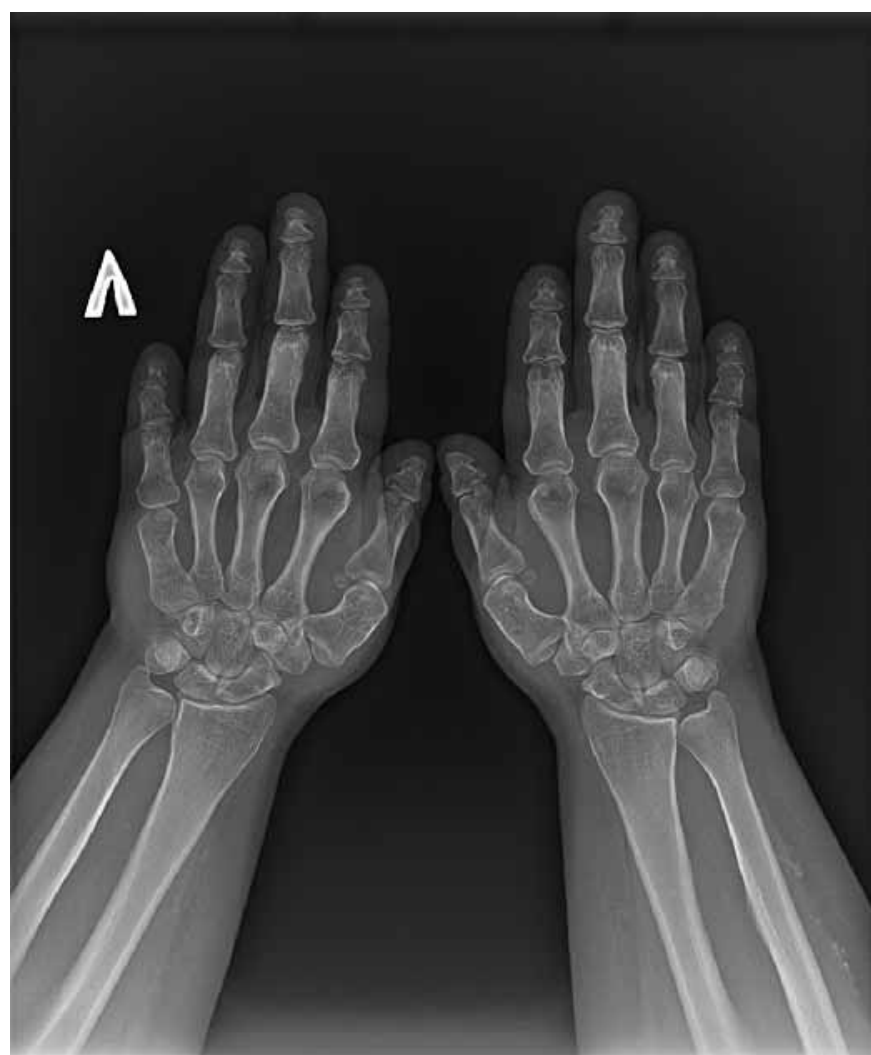

Рис. 1. Брахидактилия: выраженное укорочение V пястной кости слева.

ми расстройствами, нарушениями кальций-фосфорного обмена, гипотиреозом. С 3 лет у пациентки отмечались тонические судороги на фоне фебрильной лихорадки и периодические миалгии. Впервые обследование проведено в 12 лет в связи с появлением карпопедальных спазмов, когда была выявлена гипокальциемия (Са общ. 1,3, при норме 2,0-2,6 ммоль/л), гиперфосфатемия (2,66 ммоль/л при норме 0,7-1,6) на фоне повышенного уровня ПТГ (268 пг/мл). Во время обследования в стационаре также впервые обратили внимание на особенности фенотипа: у девочки отмечалось ожирение, укорочение IV и V пястных и плюсневых костей (рис. 1, 2), пальпировались плотные подкожные образования, не спаянные с окружающей тканью, безболезненные. Гистологическое исследование биоптата образований доказало наличие подкожных кальцификатов с распространением в перимизий. По данным мультиспиральной компьютерной томографии (МСКТ) головы выявлено обызвествление базальных ядер и субкортикальных отделов головного мозга (рис. 3). Был установлен диагноз «псевдогипопаратиреоз la типа». Молекулярно-генетическое исследование подтвердило наличие мутации в гене GNAS - гетерозиготная мутация GNAS в сайте сплайсинга с.585+2T>C. По поводу резистентности к ПтГ назначен альфакальцидол, в последующем к терапии были добавлены препараты кальция. Прием препаратов осуществлялся под контролем показателей кальция крови с периодической коррекцией дозы. В 20 лет впервые выявлен субклинический гипотиреоз и назначена заместительная терапия препаратом левотироксина натрия с дальнейшей коррекцией дозы в зависимости от уровня ТТГ при контроле в динамике. Клинических признаков гипогонадизма не отмечалось: менархе в 12 лет, менструальный цикл регулярный. При сборе семейного анамнеза не было выявлено особенностей, указывающих на возможность наличия у родственников НОО

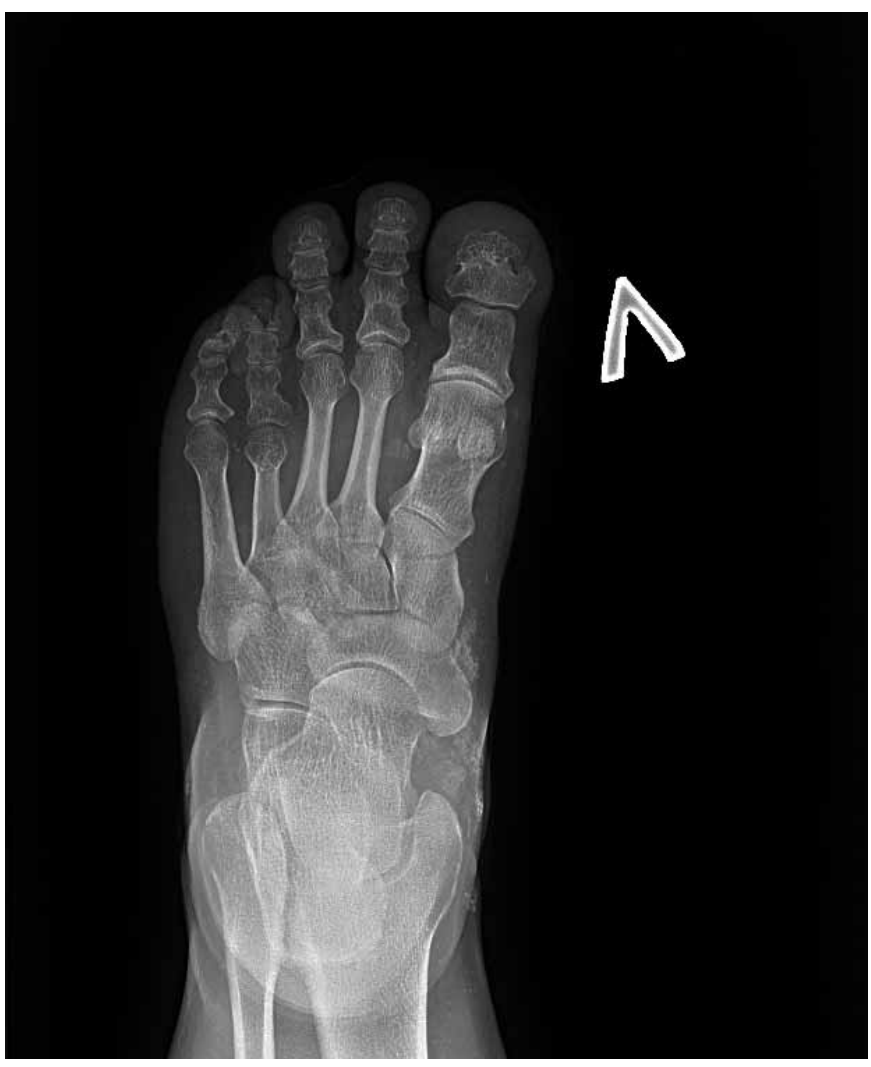

Рис. 2. Брахидактилия: укорочение IV плюсневой кости слева.

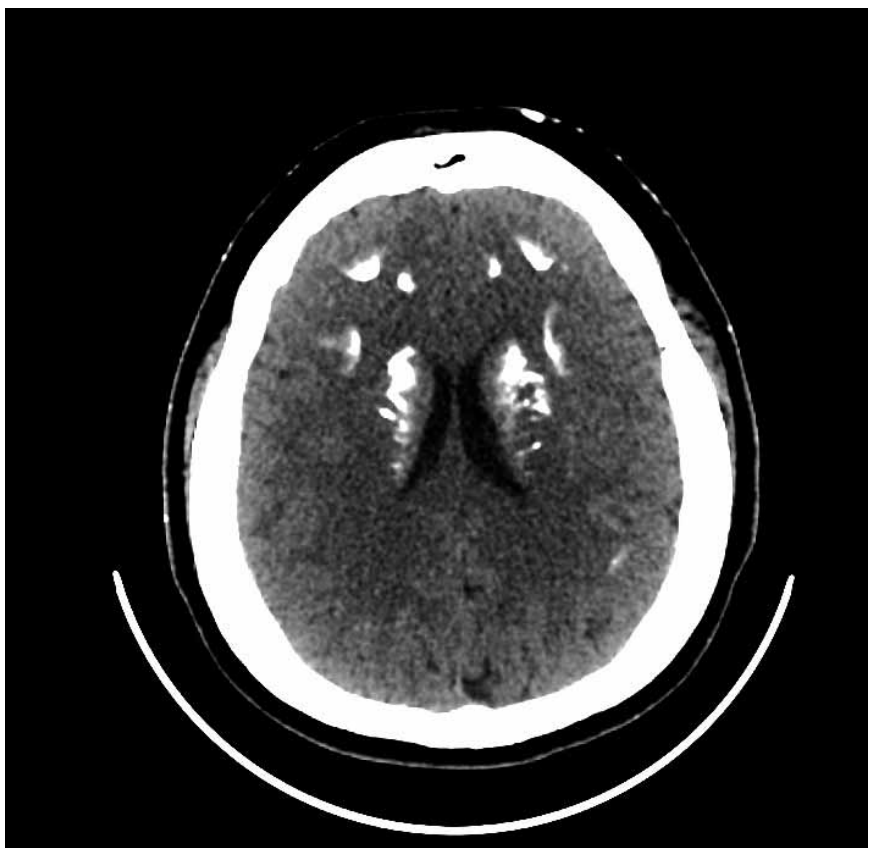

Рис. 3. Отложение кальцинатов в базальных ганглиях и субкортикальных отделах.

или гипокальциемии: родители нормального роста, брахидактилии, подкожных образований у них не имеется, судорожных эпизодов или криптогенной эпилепсии ни у родителей, ни у ближайших родственников не отмечалось. У пациентки нет детей, но было 4 беременности, все окончившиеся абортами. Одна беременность - замершая, один аборт по желанию пациентки, 2 последние беременности были прерваны после получения результатов пренатальной диагностики, доказавшей наличие у плода той же мутации в гене GNAS, что и у матери.

В 35 лет пациентка поступила в НМИЦ эндокринологии с жалобами на судорожный синдром. При осмотре обращали 


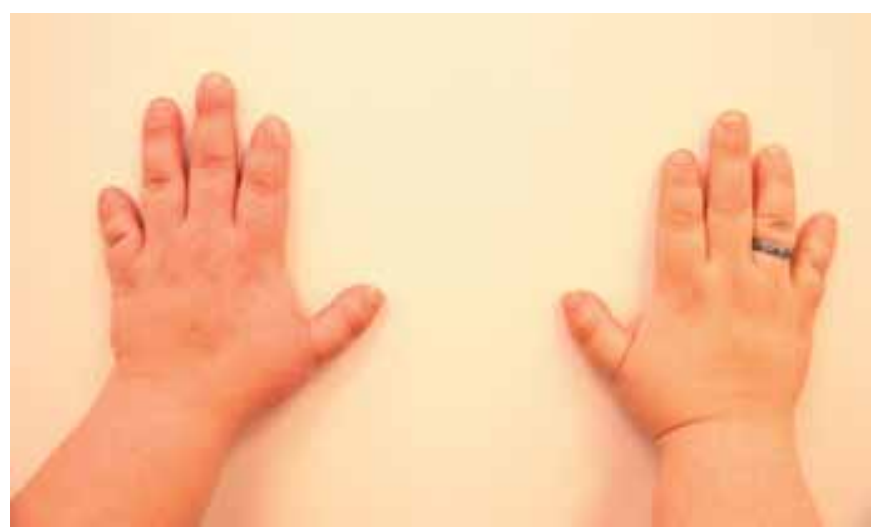

Рис. 4. Брахидактилия, фото кистей пациентки

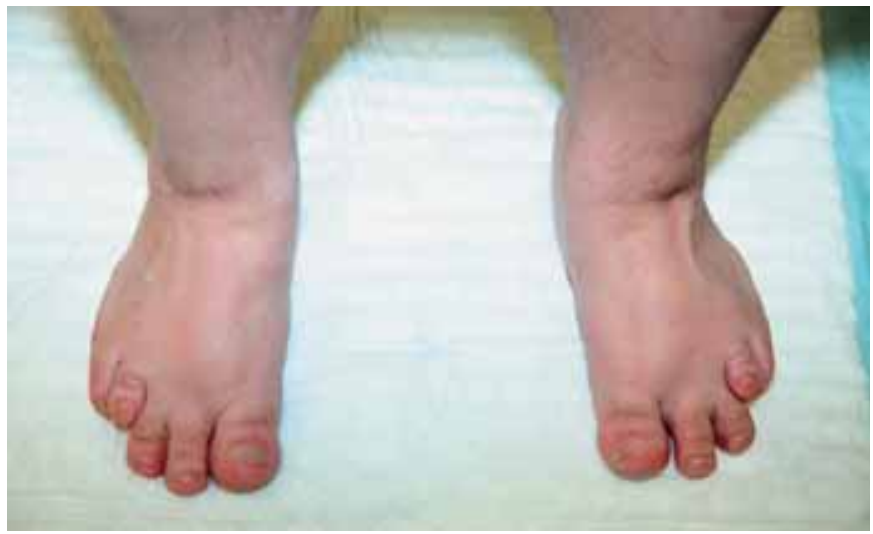

Рис. 5. Брахидактилия, фото стоп пациентки

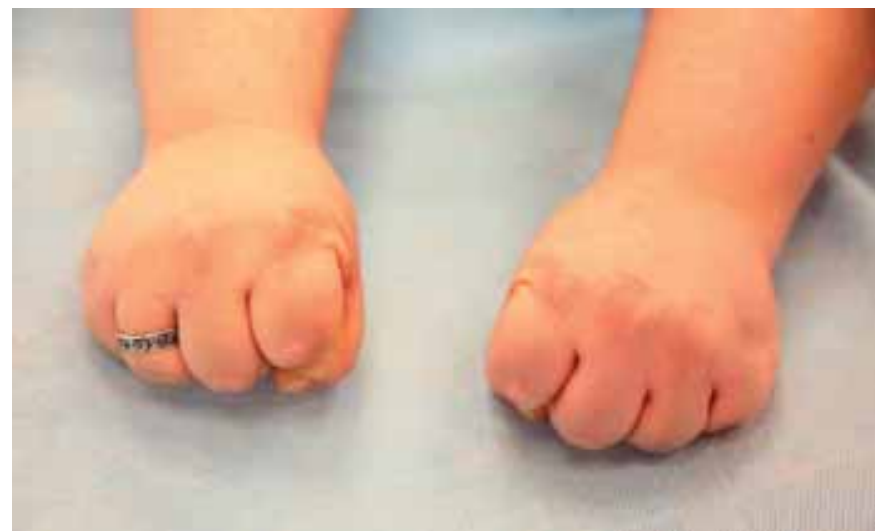

Рис. 6. Брахиметафалангизм - сглаженность в области IV-V пястно-фаланговых суставов.

на себя внимание низкорослость (рост 148 см), ожирение (87 кг, ИМТ 39) с лунообразным лицом, брахидактилия (рис. 4, 5), подкожные кальцинаты, положительный симптом Хвостека. При сжатии кистей в кулак отмечалась сглаженность в области IV-V пястно-фаланговых суставов - брахиметафалангизм (рис. 6). В ходе обследования была выявлена декомпенсация по кальций-фосфорному обмену и гипотиреозу: гипокальциемия (Са ион. 0,87 ммоль/л при норме 1,03-1,29), гиперфосфатемия (1,81 ммоль/л при норме 0,74-1,52), повышение уровня ПТГ до 136 пг/мл (15-65), гипотиреоз (ТТГ 7,37 мМЕ/л при норме 0,64-5,76, св.Т4 8,72 пмоль/л при норме 11,5-20,4) при нормальном уровне аутоантител (АТ) (АТ к тиреопероксидазе (ТПО) 1,77 MЕ/мл, при норме 0-5,6) и отсутствии эхо-признаков аутоиммунного тиреоидита по данным УзИ щитовидной железы (рис. 7). В суточном анализе мочи гипокальциурия 0,804 ммоль/сут (2,5-8), 0,37 мг/ кг/сут, гипофосфатурия - 12,52 ммоль/сут (13-42). Беседа с пациенткой выявила ряд психологических проблем, появ-

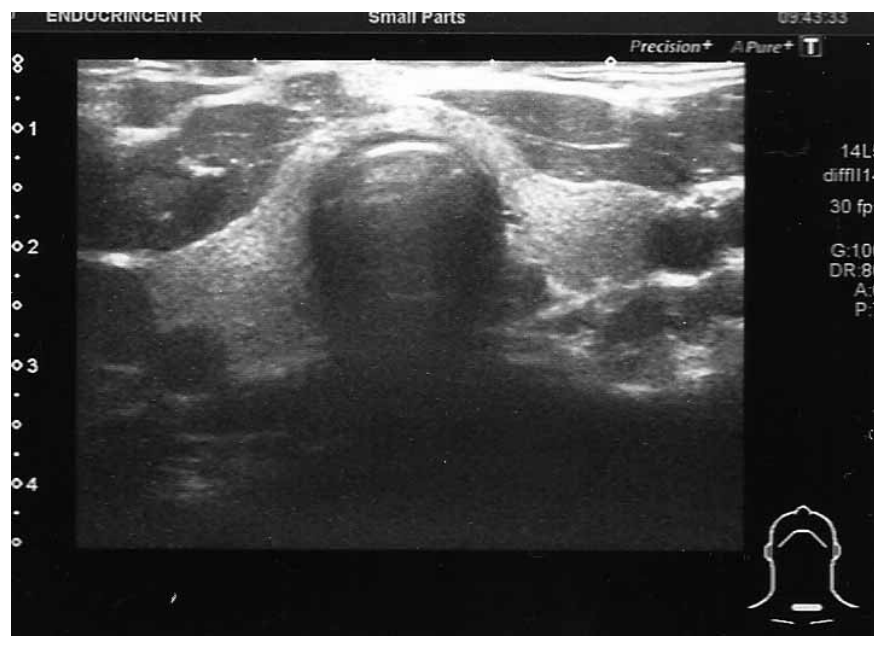

Рис. 7. Отсутствие эхографических признаков аутоиммунного поражения щитовидной железы

ление которых сама женщина связывала с тяжелым переживанием последнего аборта: на фоне депрессии перестала следить за питанием и регулярным приемом препаратов, отмечались прогрессирующий набор веса и периодическая боль в мышцах, развившаяся в итоге в карпопедальный спазм. Проявления острой гипокальциемии были купированы введением раствора кальция глюконата; показатели кальций-фосфорного обмена и функционального статуса щитовидной железы нормализовались после восстановления и коррекции приема альфакальцидола и левотироксина натрия. По поводу имеющейся депрессии получает антидепрессанты под наблюдением психоневролога.

Таким образом, на основании данных за резистентность к ПТГ (гипокальциемия, гиперфосфатемия на фоне повышенного ПТГ) в сочетании с резистентностью к ТТГ (первичный гипотиреоз без признаков аутоиммунного поражения) и фенотипическими признаками НОО (подкожные кальцинаты, брахидактилия, низкорослость, ожирение, лунообразное лицо) был установлен диагноз: псевдогипопаратиреоз Іа типа, осложненный синдромом Фара, подтвержденный молекулярно-генетически.

\section{ОБСУЖДЕНИЕ}

Представленный клинический случай является ярким примером редкого состояния множественной гормональной резистентности, метаболических нарушений и классическим вариантом ПГП: пациентке на основании сочетания полного фенотипа НОО с признаками нечувствительности к ПТГ был установлен ПГП типа Іа. Такое название заболевания относится к исторически сложившейся, но все еще используемой классификации. В силу особенностей экспрессии GNAS проявления его варьируют от изолированной ПТГ-резистентности, что принято было обозначать как ПГП типа Ib, до полного фенотипа НОO c мультигормональной резистентностью, обозначаемого как раз как ПГП типа Іа. Кроме того, выделяют ПГП Іс, при котором имеется частично сохранная активность Gas и ПГП II типа и нет дефектов в гене GNAS. В настоящее время обсуждается новая классификация, объединяющая в себе все возможные инактивирующие нарушения передачи сигнала от ПТГ-рецептора к клеточному ядру. Согласно новой классификации, не получившей пока широкого распространения, в описываемом случае ПГП определяется 
как заболевание, обусловленное инактивацией ПтГ-сигнального пути 2 типа (inactivating РTH/PTHrP signalling disorder, iPPSD2). Патогенная мутация в гене GNAS обусловила нарушение функции Gas и привела к формированию фенотипических особенностей, включающих низкорослость, брахидактилию, подкожные кальцинаты и ожирение, а также обусловила нарушение кальций-фосфорного обмена вследствие нечувствительности к ПтГ и развитие гипотиреоза за счет резистентности к ТТГ.

Исходя из данных анамнеза, можно предположить у пациентки длительно нерегистрируемую гипокальциемию: не исключено, что миалгии и тонические судороги, которыми пациентка страдала с 3 лет, были обусловлены низким уровнем кальция крови. Показательно, что данные жалобы не расценивались как основание для исследования кальций-фосфорного обмена вплоть до возникновения более выраженных клинических проявлений в виде карпопедальных спазмов. У данной пациентки ПГП мог быть заподозрен раньше не столько даже на основании проявлений резистентности к ПТГ, сколько на основании наличия брахидактилии и подкожных кальцинатов специфических признаков заболевания, обусловленного инактивацией ПТГ-сигнального пути. Гипокальциемия при ПГП купируется назначением альфакальцидола или кальцитриола, что обусловлено патогенезом заболевания: резистентность к ПТГ приводит к сниженной активности 1-альфа-гидроксилазы, нарушению образования активной формы витамина D и, как следствие, гипокальциемии. Ежедневный прием подобранной дозы и регулярный контроль уровня кальция крови достаточны для своевременной коррекции терапии и предупреждения гипокальциемии. Имевшаяся у пациентки тяжелая декомпенсация по кальций-фосфорному обмену была обусловлена низкой приверженностью к лечению и пропусками в терапии.

На момент диагностики заболевания в 12 лет уже имелось обызвествление базальных ядер и субкортикальных отделов головного мозга, отражающих наличие синдрома Фара. Синдром Фара является неспецифическим осложнением длительной гиперфосфатемии и гипокальциемии, ведущих к отложению солей кальция в тканях головного мозга и хрусталике. Считается, что отложение кальцинатов в структурах головного мозга приводит к развитию нейропсихических нарушений в виде снижения памяти, концентрации внимания и нарушений экстрапирамидной системы в виде дистонии и расстройств непроизвольных движений, что обосновано характером локализации кальцинатов. В статьях, посвященных редким вариантам психических нарушений, у пациентов с ПГП основой психических расстройств называют именно синдром Фара. Тем не менее нельзя считать синдром Фара ведущей причиной психических нарушений: одним из характерных признаков НОО является умственная отсталость, манифестирующая, как правило, с первых лет жизни, ее развитие обусловлено влиянием нарушенного внутриклеточного сигналинга и сниженной активностью Gas и наблюдается вне зависимости от синдрома Фара. Кроме того, наличие синдрома Фара не обязательно будет иметь клинические проявления. Несмотря на длительное существование кальцинатов в экстрапирамидных структурах, у нашей пациентки не было выявлено характерных неврологических нарушений, а имеющаяся депрессия могла быть не столько проявлением синдрома Фара, сколько компонентом HOO, отражающим нарушение активности
Gas, либо мог иметься экзогенный компонент: сама пациентка связывала свое тяжелое психоэмоциональное состояние со стрессом после перенесенного последнего аборта.

Помимо депрессивных состояний и гипокальциемии, наиболее клинически значимым и сказывающимся на качестве жизни компонентом заболевания у пациентки было ожирение. Развитие ожирения при ПГП связано не с нарушенным пищевым поведением и гиперфагией, а с низким расходом энергии в покое и ассоциировано со сниженной активностью симпатической нервной системы и нарушенной липолитической активностью эпинефрина. Специфического подхода к снижению веса при ПГП не разработано, рекомендации сводятся к снижению калорийности питания, увеличению двигательной активности и физических нагрузок. В нашем случае достигнуть снижения веса пациентке было сложно из-за депрессии, обусловившей низкую комплаентность к заместительной терапии и неготовность к изменению образа жизни.

\section{ЗАКЛЮЧЕНИЕ}

Псевдогипопаратиреоз - редкое многокомпонентное заболевание, в котором специфические проявления, такие как резистентность к ПТГ, подкожные кальцинаты и брахидактилия, сочетаются с неспецифическими, но характерными составляющими в виде ожирения, низкорослости и психоневрологических расстройств.

Ведущими проявлениями метаболических расстройств при ПГП являются нарушение кальций-фосфорного обмена в виде гипокальциемии и синдрома Фара, а также ожирение. ПГП часто выявляется на основании обследования по поводу судорожного синдрома, но идентификация специфических компонентов НОО может помочь вовремя заподозрить и диагностировать заболевание до развития клинических проявлений резистентности к ПТГ.

Хотя диагноз устанавливается на основании клинической картины и лабораторного исследования и при тщательном сборе семейного анамнеза возможно выявление родственников с ПГП, проведение молекулярно-генетического исследования необходимо для медико-генетического консультирования семьи.

Синдром Фара с отложением кальцинатов в структурах экстрапирамидной системы не всегда приводит к развитию двигательных нарушений.

Психические расстройства в виде депрессии могут усугубить тяжесть состояния, ведя к декомпенсации по гормональной резистентности и прогрессированию ожирения.

Осведомленность врачей о вариантах манифестации и спектре возможных компонентов ПГП способна улучшить раннюю диагностику заболевания, предотвратить осложнения и определить индивидуальную тактику ведения с учетом вариабельности компонентов.

\section{ДОПОЛНИТЕЛЬНАЯ ИНФОРМАЦИЯ}

Согласие пациента. Пациентка добровольно подписала информированное согласие на публикацию персональной медицинской информации в обезличенной форме.

Конфликт интересов. Авторы декларируют отсутствие явных и потенциальных конфликтов интересов, связанных с публикацией настоящей статьи. 


\section{СПИСОК ЛИТЕРАТУРЫ | REFERENCES}

1. Albright F, Burnett, C, Smith, P. H, Parson, W. Pseudo-hypoparathyroidism--an example of 'Seabright-Bantam syndrome': report of three cases. Endocrinology. 1942;30:922-32.

2. Levine MA, Downs RW, Jr., Singer $M$, et al. Deficient activity of guanine nucleotide regulatory protein in erythrocytes from patients with pseudohypoparathyroidism. Biochem. Biophys. Res. Commun. 1980;94(4):1319-1324

3. Patten $J$, Johns DR, Valle D, et al. Mutation in the Gene Encoding the Stimulatory G Protein of Adenylate Cyclase in Albright's Hereditary Osteodystrophy. N. Engl. J. Med. 1990;322(20):1412-1419. doi: 10.1056/ nejm199005173222002.

4. Elli FM, Linglart A, Garin I, et al. The Prevalence ofGNASDeficiency-Related Diseases in a Large Cohort of Patients Characterized by the EuroPHP Network. J. Clin. Endocr. Metab. 2016;101(10):3657-3668. doi: 10.1210/jc.2015-4310.

5. Fernández-Rebollo E, Lecumberri B, Gaztambide S, et al. Endocrine Profile and Phenotype-(Epi)Genotype Correlation in Spanish Patients with Pseudohypoparathyroidism. J. Clin. Endocr. Metab. 2013;98(5):E996-E1006. doi: 10.1210/jc.2012-4164.

6. Sano S, Nakamura A, Matsubara K, et al. (Epi)genotype-Phenotype Analysis in 69 Japanese Patients With Pseudohypoparathyroidism Type I. Journal of the Endocrine Society. 2018;2(1):9-23. doi: 10.1210/ js.2017-00293.

7. Mantovani G. Pseudohypoparathyroidism: Diagnosis and Treatment. J. Clin. Endocr. Metab. 2011:96(10):3020-3030. doi: 10.1210/jc.2011-1048.

8. Thiele S, Mantovani G, Barlier A, et al. From pseudohypoparathyroidism to inactivating PTH/PTHrP signalling disorder (iPPSD), a novel classification proposed by the EuroPHP network. European journal of endocrinology / European Federation of Endocrine Societies. 2016;175(6):P1-P17. doi: 10.1530/eje-16-0107.
9. Saleem S, Aslam H, Anwar M, et al. Fahr's syndrome: literature review of current evidence. Orphanet J. Rare Dis. 2013;8(1):156. doi 10.1186/1750-1172-8-156.

10. Otheman Y, Khalloufi H, Benhima I, Ouanass A. Manifestations neuropsychiatriques révélant une pseudohypoparathyrö̈die avec un syndrome de Fahr. L'Encéphale. 2011;37(1):54-58. doi: 10.1016/j.encep.2010.03.001

11. Farfel Z, Friedman E. Mental deficiency in pseudohypoparathyroidism type I is associated with Ns-protein deficiency. Ann. Intern. Med. 1986;105(2):197-199

12. Mouallem M, Shaharabany M, Weintrob N, et al. Cognitive impairment is prevalent in pseudohypoparathyroidism type la, but not in pseudopseudohypoparathyroidism: possible cerebral imprinting of Gs? Clin. Endocrinol. (Oxf.). 2007:0(0):070907132242011-??? doi: 10.1111/j.1365-2265.2007.03025.x

13. Perez KM, Lee EB, Kahanda S, et al. Cognitive and behavioral phenotype of children with pseudohypoparathyroidism type 1A. Am. J. Med. Genet. A. 2018;176(2):283-289. doi: 10.1002/ajmg.a.38534.

14. Chen M, Berger A, Kablan A, et al. Gsa Deficiency in the Paraventricular Nucleus of the Hypothalamus Partially Contributes to Obesity Associated with Gsa Mutations. Endocrinology. 2012;153(9):4256-4265. doi: 10.1210/en.2012-1113.

15. Roizen JD, Danzig J, Groleau V, et al. Resting Energy Expenditure Is Decreased in Pseudohypoparathyroidism Type 1A. J. Clin. Endocr. Metab. 2016;101(3):880-888. doi: 10.1210/jc.2015-3895.

16. 1Carel JC, Le Stunff C, Condamine L, et al. Resistance to the Lipolytic Action of Epinephrine: A New Feature of Protein GsDeficiency. J. Clin Endocr. Metab. 1999;84(11):4127-4131. doi: 10.1210/jcem.84.11.6145.

17. Chen M, Shrestha YB, Podyma B, et al. Gsa deficiency in the dorsomedial hypothalamus underlies obesity associated with Gsa mutations. J. Clin. Invest. 2016;127(2):500-510. doi: 10.1172/jci88622.

\section{ИНФОРМАЦИЯ ОБ АВТОРАХ [AUTHORS INFO]}

*Дзеранова Лариса Константиновна, д.м.н. [Larisa K. Dzeranova, ScD]; адрес: Россия, 117036, Москва, ул. Дм. Ульянова, д. 11 [address: 11 Dm. Ulyanova street, 117036 Moscow, Russia]; ORCID: http://orcid.org/0000-0002-0327-4619; eLibrary SPIN: 2958-5555; e-mail: dzeranovalk@yandex.ru

Маказан Надежда Викторовна, педиатр-эндокринолог [Nadezhda V. Makazan, MD];

ORCID: http://orcid.org/0000-0003-3832-6267; eLibrary SPIN 7156-6517; e-mail: nmakazan@yandex.ru.

Пигарова Екатерина Александровна, К.М.н. [Ekaterina A. Pigarova, PhD];

ORCID: http://orcid.org/0000-0001-6539-466X; eLibrary SPIN: 6912-6331; e-mail: kpigarova@gmail.com.

Тюлякова Анна Николаевна, ординатор [Anna N. Tyulyakova, residence]; ORCID: http://orcid.org/0000-0002-2455-6383; eLibrary SPIN: 7717-9220;e-mail: anya_93_08@mail.ru.

Артемова Екатерина Викторовна, врач [Ekaterina V. Artemova, MD]; ORCID: http://orcid.org/ 0000-0002-2232-4765; eLibrary SPIN: 4649-0765; e-mail: profilaktika@bk.ru.

Солдатова Татьяна Васильевна, К.м.н. [Tatiana V. Soldatova, PhD]; ORCID: http://orcid.org/0000-0003-1904-3118; e-mail: tatmoscow@yandex.ru.

Тулупов Денис Олегович [Denis О. Tulupov]; ORCID: http://orcid.org/0000-0001-7812-0545; eLibrary SPIN: 9844-7885; e-mail: denis.tylypov@mail.ru.

Воронцов Александр Валерьевич, д.м.н. [Aleksandr V. Vorontsov, ScD]; ORCID: http://orcid.org/0000-0002-2791-3278; eLibrary SPIN: 2502-4463; e-mail:mr2005i@mail.ru.

Мельниченко Галина Афанасьевна, акад. PAH [Galina A. Melnichenko, academician of RAS, ScD, Professor]; ORCID: http://orcid.org/0000-0002-5634-7877; eLibrary SPIN: 8615-0038; e-mail: teofrast2000@mail.ru

\section{ЦИТИРОВАТЬ:}

Дзеранова Л.К., Маказан Н.В., Е.А. Пигарова, Тюлякова А.Н., Артемова Е.В., Солдатова Т.В., Тулупов Д.О., Воронцов А.В., Мельниченко Г.А. Множественная гормональная резистентность и метаболические нарушения при псевдогипопаратиреозе // Ожирение и метаболизм. — 2018. — Т.15. — № 2 - C. 51-55. doi: 10.14341/OMET20182

\section{TO CITE THIS ARTICLE:}

Dzeranova LK, Makazan NV, Pigarova EA, Tyulyakova AN, Artemova EV, Soldatova TV, Tulupov DO, Vorontsov AV, Melnichenko GA. Multiple hormonal resistance and metabolic disorders in pseudogypoparatiosis. Obesity and metabolism. 2018;15(2):51-55. doi: 10.14341/OMET20182 